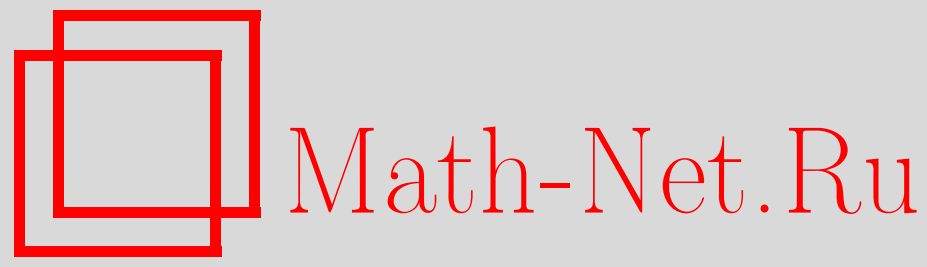

А. А. Аграчев, О пространствах симметричных операторов с кратными основными состояниями, Функи. анализ и его прил., 2011, том 45, выпуск 4, 1-15

DOI: https://doi.org/10.4213/faa3049

Использование Общероссийского математического портала MathNet.Ru подразумевает, что вы прочитали и согласны с пользовательским соглашением http://www . mathnet.ru/rus/agreement

Параметры загрузки:

IP : 44.207 .124 .84

26 апреля 2023 г., 12:22:27

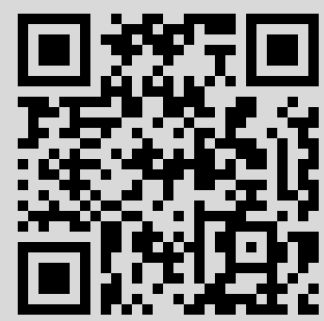


Функционалъный анализ и его приложения

2011, т. 45, вып. 4, с. 1-15

УДК $515.146+512.64$

\title{
О пространствах симметричных операторов с кратными основными состояниями
}

\author{
(C) 2011. A. A. АГРАЧЕВ
}

Памяти В. И. Арнолъда

Исследуется гомологическая структура фильтрации пространства самосопряженных операторов по кратности основного состояния. Рассматриваются только операторы, действующие в конечномерном комплексном или вещественном гильбертовом пространстве, но бесконечномерные обобщения легко угадываются.

\section{$\S 1$. Введение}

Статья посвящается памяти Владимира Игоревича Арнольда и, можно сказать, вдохновлена его известными работами [1], [2] (см. также статью [5]). Она открывает запланированную серию статей о гомологических инвариантах семейств квадратичных форм и сходных геометрических объектов; доказанная ниже теорема 2 служит фундаментом всего того, что последует.

В этой статье изучается фильтрация пространства самосопряженных операторов по кратности основного состояния. Мы ограничиваемся операторами в конечномерном комплексном или вещественном гильбертовом пространстве, но возможные бесконечномерные обобщения легко угадываются.

Пусть $\lambda_{1}(A) \leqslant \lambda_{2}(A) \leqslant \cdots \leqslant \lambda_{k}(A) \leqslant \cdots-$ упорядоченные собственные числа оператора $A$. Операторы, обладающие основным состоянием кратности не менее $k$, характеризуются уравнением $\lambda_{1}(A)=\lambda_{k}(A)$. Теорема 1 описывает гомотопический тип пространства нетривиальных решений этого уравнения: оно оказывается гомотопически эквивалентным пространству Тома определенного векторного расслоения над некоторым грассманианом.

Рост кратности от $k$ к $k+1$ реализуется пересечением пространства решений уравнения $\lambda_{1}(A)=\lambda_{k}(A)$ с решениями уравнения $\lambda_{k}(A)=\lambda_{k+1}(A)$. Мы изучаем гомологическую структуру этой операции пересечения.

Как обычно, удобнее принять двойственную точку зрения, т. е. иметь дело с когомологиями пары $\left(\mathbb{B},\left\{A \in \mathbb{B}: \lambda_{1}(A) \neq \lambda_{k}(A)\right\}\right)$ вместо гомологий пространства $\left\{A \in \mathbb{B}: \lambda_{1}(A)=\lambda_{k}(A)\right\}$, где $\mathbb{B}$ - пространство всех самосопряженных операторов. При этом пересечение циклов заменяется обычным когомологическим умножением.

Пространство решений уравнения $\lambda_{k}(A)=\lambda_{k+1}(A)$ есть цикл коразмерности 3 в комплексном случае и цикл по модулю 2 коразмерности 2 в вещественном случае. Двойственный объект есть трехмерный класс когомологий в комплексном и двумерный класс когомологий по модулю 2 в вещественном случае; имеется в виду класс когомологий пары $\left(\mathbb{B},\left\{A \in \mathbb{B}: \lambda_{k}(A) \neq \lambda_{k+1}(A)\right\}\right)$. 
В обоих случаях мы обозначаем этот когомологический класс символом $\Gamma_{k}$ и изучаем отображение когомологий пары $\left(\mathbb{B},\left\{A \in \mathbb{B}: \lambda_{1}(A) \neq \lambda_{k}(A)\right\}\right)$ в когомологии пары $\left(\mathbb{B},\left\{A \in \mathbb{B}: \lambda_{1}(A) \neq \lambda_{k+1}(A)\right\}\right)$, переводящее каждый когомологический класс в его произведение с $\Gamma_{k}$. Главный результат статьи, теорема 2 , утверждает, что последовательность этих отображений для $k=1,2, \ldots$ есть точная последовательность.

Рассмотрим простейший случай самосопряженных операторов на $\mathbb{C}^{2}$ или, иными словами, эрмитовых $2 \times 2$-матриц. Пара $\left(\mathbb{B},\left\{A \in \mathbb{B}: \lambda_{1}(A) \neq \lambda_{k}(A)\right\}\right)$ равна $\left(\mathbb{R}^{4}, \varnothing\right)$ при $k=1$ и $\left(\mathbb{R}^{4}, \mathbb{R}^{4} \backslash \mathbb{R}\right)$ при $k=2$. Точная последовательность из теоремы 2 сводится к очевидной последовательности

$$
0 \rightarrow H^{*}\left(\mathbb{R}^{3}\right) \rightarrow H^{*+3}\left(\mathbb{R}^{3}, \mathbb{R}^{3} \backslash 0\right) \rightarrow 0 .
$$

Общее многомерное вычисление отнюдь не тривиально и, похоже, имеет фундаментальную природу, что мы надеемся показать в последующих публикациях.

В следующем параграфе вводятся нужные обозначения и напоминаются известные факты о пространствах самосопряженных операторов. Теоремы 1 и 2 сформулированы и доказаны в $\S 3$.

Все пары топологических пространств и их подпространств, с которыми мы имеем дело, гомотопически эквивалентны парам конечных клеточных комплексов и их подкомплексов. Пусть $(M, X),(M, Y)$ и $(M, X \cup Y)$ - такие пары, $\xi \in H^{i}(M, X), \eta \in H^{j}(M, Y)$; тогда $\xi \smile \eta \in H^{i+j}(M, X \cup Y)$ есть когомологическое произведение элементов $\xi$ и $\eta$.

\section{§2. Предварительные сведения}

Мы рассматриваем пространства самосопряженных операторов на $\mathbb{R}^{n}$ или $\mathbb{C}^{n}$. В обоих случаях через $\lambda_{1}(A) \leqslant \cdots \leqslant \lambda_{n}(A)$ обозначаются упорядоченные собственные числа данного оператора $A$. Пусть $I$ - единичный оператор, а $\alpha$ - положительное вещественное число; тогда $\lambda_{i}(A \pm \alpha I)=\lambda_{i}(A) \pm \alpha, \lambda_{i}(\alpha A)=\alpha \lambda_{i}(A)$. Кроме того, $A \pm \alpha I$ и $\alpha A$ имеют те же собственные векторы, что $A$. Удобно не различать операторы, получаемые один из другого только что описанными простыми преобразованиями.

Обозначим через $\mathbb{S}(\mathbb{R})$ (соответственно $\mathbb{S}(\mathbb{C})$ ) пространство всех нескалярных самосопряженных линейных операторов $A: \mathbb{R}^{n} \rightarrow \mathbb{R}^{n}$ (соответственно $A: \mathbb{C}^{n} \rightarrow$ $\left.\mathbb{C}^{n}\right)$, факторизованное по отношению эквивалентности $A \sim(\alpha A+\beta I)$ для любых $\alpha>0, \beta \in \mathbb{R}$. Тогда $\mathbb{S}(\mathbb{R})$ гомеоморфно сфере $S^{n(n+1) / 2-2}$, а $\mathbb{S}(\mathbb{C})$ гомеоморфно $S^{n^{2}-2}$. В дальнейшем мы будем одновременно работать с вещественным и эрмитовым случаями и будем просто опускать аргумент у $\mathbb{S}$. Конус над $\mathbb{S}$ обозначим символом $\mathbb{B}$; это шар размерности $n(n+1) / 2-1$ в вещественном случае и шар размерности $n^{2}-1$ в эрмитовом случае.

Факторизацию можно заменить нормализацией и определить $\mathbb{S}$ как пространство таких самосопряженных операторов $A$, что $\sum_{i=1}^{n} \lambda_{i}(A)=0, \sum_{i=1}^{n} \lambda_{i}^{2}(A)=1$; тогда $\mathbb{B}$ определяется соотношениями $\sum_{i=1}^{n} \lambda_{i}(A)=0, \sum_{i=1}^{n} \lambda_{i}^{2}(A) \leqslant 1$. В одних случаях удобнее факторизация, а в других нормализация, и мы часто используем один и тот же символ для обозначения класса эквивалентности и его представителя; это упрощает обозначения и не приводит к путанице. 
Рассмотрим открытые подмножества

$$
\Sigma_{k, k+1} \doteq\left\{A \in \mathbb{S}: \lambda_{k}(A) \neq \lambda_{k+1}(A)\right\} .
$$

Хорошо известны следующие свойства этих подмножеств:

Предложение $1 . \mathbb{S} \backslash \Sigma_{k, k+1}$ - алгебраическое подмножсество в $\mathbb{S}$ коразмерности 2 в вещественном и коразмерности 3 в эрмитовом случае. Особъе точки алгебраического множсества $\mathbb{S} \backslash \Sigma_{k, k+1}$ суть операторь с не менее чем трехкратным собственным числом $\lambda_{k}$; они составляют алгебраическое подмножество в $\mathbb{S}$ коразмерности 5 в вещественном и коразмерности 8 в эрмитовом случае. Кроме того, регулярная часть подмножества $\mathbb{S} \backslash \Sigma_{k, k+1}$ ориентируема в эрмитовом случае.

Набросок доказательства. Пусть $A_{0} \in \mathbb{S} \backslash \Sigma_{k, k+1}$, а $J_{A_{0}}$ - множество всех таких $j \in\{1, \ldots, n\}$, что $\lambda_{j}\left(A_{0}\right)=\lambda_{k}\left(A_{0}\right)$; тогда \# $J_{A_{0}} \geqslant 2$. Для данного самосопряженного оператора $A$ положим

$$
K_{A}=\operatorname{span}\left\{x \in X: A x=\lambda_{j}(A) x, j \in J_{A_{0}}\right\},
$$

где $X$ - это $\mathbb{R}^{n}$ в вещественном и $\mathbb{C}^{n}$ в эрмитовом случае.

Пусть $\mathscr{O}_{0}$ - такая окрестность оператора $A_{0}$ в $\mathbb{S}$, что $K_{A} \cap K_{A_{0}}^{\perp}=0$ для любого $A \in \mathscr{O}_{0}$. Обозначим через $P_{A_{0} A}^{k}: K_{A} \rightarrow K_{A_{0}}$ ограничение на $K_{A}$ ортогонального проектора $X \rightarrow K_{A_{0}}$ и положим $\Phi(A)=P_{A_{0} A} A P_{A_{0} A}^{-1}, A \in \mathscr{O}_{0}$. Тогда $\Phi$ - корректно определенное рациональное отображение окрестности $\mathscr{O}_{0}$ в пространство самосопряженных операторов на $K_{A_{0}}$. Дифференциал отображения $\Phi$ в точке $A_{0}$ отображает $A$ в композицию оператора $\left.A\right|_{K_{A_{0}}}$ с ортогональной проекцией $X \rightarrow K_{A_{0}}$. В частности, линейное отображение $D_{A_{0}} \Phi$ сюръективно; следовательно, $\Phi$ - субмерсия на некоторой окрестности точки $A_{0}$. Можно предположить, что $\Phi$ - субмерсия на всей окрестности $\mathscr{O}_{0}$. Кроме того, $\lambda_{i}(\Phi(A))=\lambda_{i+j_{0}}(A), i=1, \ldots, \# J_{A_{0}}$, где $j_{0}=\min J_{A_{0}}$.

Пусть $A \in \mathscr{O}_{0} ;$ равенство $J_{A}=J_{A_{0}}$ имеет место в том и только том случае, когда $\Phi(A)$ - скалярный оператор. Следовательно, $\left\{A \in \mathscr{O}_{0}: J_{A}=J_{A_{0}}\right\}-$ регулярное алгебраическое подмножество в $\mathscr{O}_{0}$ коразмерности $j_{0}\left(j_{0}+1\right) / 2-1$ в вещественном и $j_{0}^{2}-1$ в эрмитовом случае.

Осталось доказать ориентируемость в эрмитовом случае. Достаточно показать, что пространство самосопряженных операторов на $K_{A}, A \in \mathbb{S}$, обладает канонической ориентацией. Ориентация пространства самосопряженных операторов индуцирована ориентацией самого пространства $K_{A}$, а ориентация пространства $K_{A} \subset \mathbb{C}^{n}$ определена комплексной структурой (любое комплексное пространство обладает канонической ориентацией).

Из предложения 1 следует, что $H_{\operatorname{dim} \mathbb{S}-2}\left(\mathbb{S} \backslash \Sigma_{k, k+1} ; \mathbb{Z}_{2}\right)=\mathbb{Z}_{2}$ в вещественном и $H_{\operatorname{dim} \mathbb{S}-3}\left(\mathbb{S} \backslash \Sigma_{k, k+1} ; \mathbb{Z}\right)=\mathbb{Z}$ в эрмитовом случае. Согласно двойственности Александера, $H^{1}\left(\Sigma_{k, k+1} ; \mathbb{Z}_{2}\right)=\mathbb{Z}_{2}$ в вещественном случае, причем значение образующей группы $H^{1}\left(\Sigma_{k, k+1} ; \mathbb{Z}_{2}\right)$ на замкнутой кривой в $\Sigma_{k, k+1}$ равно коэффициенту зацепления этой кривой с $\mathbb{S} \backslash \Sigma_{k, k+1}$ по модулю 2 . Аналогично, $H^{2}\left(\Sigma_{k, k+1} ; \mathbb{Z}\right)=\mathbb{Z}$ в эрмитовом случае, и значение образующей группы $H^{1}\left(\Sigma_{k, k+1} ; \mathbb{Z}\right)$ на компактной ориентированной поверхности в $\Sigma_{k, k+1}$ равно коэффициенту зацепления этой поверхности с $\mathbb{S} \backslash \Sigma_{k, k+1}$. 
В дальнейшем в этой статье мы всегда рассматриваем гомологии и когомологии с коэффициентами в $\mathbb{Z}_{2}$ в вещественном и с коэффициентами в $\mathbb{Z}$ в эрмитовом случае и опускаем указание на коэффициенты, чтобы упростить обозначения. Кроме того, мы обозначаем через $\varepsilon$ коразмерность подмножества $\mathbb{S} \backslash \Sigma_{k, k+1}$ в $\mathbb{S}$; таким образом, $\varepsilon=2$ в вещественном и $\varepsilon=3$ в эрмитовом случае.

Для данного $A \in \Sigma_{k, k+1}$ положим

$$
E_{A}^{k}=\operatorname{span}\left\{x \in X: A x=\lambda_{i}(A) x, i=1, \ldots, k\right\},
$$

где $X=\mathbb{R}^{n}$ в вещественном и $X=\mathbb{C}^{n}$ в комплексном случае. Тогда $\mathscr{E}^{k}=$ $\left\{(x, A): A \in \Sigma_{k, k+1}, x \in E_{A}^{k}\right\}$ есть $k$-мерное векторное подрасслоение тривиального расслоения $X \times \Sigma_{k, k+1}$ над $\Sigma_{k, k+1}$. Пусть $\gamma_{k} \in H^{\varepsilon-1}\left(\Sigma_{k, k+1}\right)-$ первый характеристический класс Штифеля-Уитни этого расслоения в вещественном и первый класс Чжэня в эрмитовом случае.

Предложение 2. $\gamma_{k}$ есть образующал группы $H^{\varepsilon-1}\left(\Sigma_{k, k+1}\right)$.

Доказательство. Нужно вычислить характеристические классы ограничения расслоения $\mathscr{E}^{k} \rightarrow \Sigma_{k, k+1}$ на $(\varepsilon-1)$-мерное компактное подмногообразие в $\Sigma_{k, k+1}$, имеющее коэффициент зацепления с $\mathbb{S} \backslash \Sigma_{k, k+1}$, равный \pm 1 .

Обозначим через $\mathbb{S}_{2}$ пространство таких самосопряженных операторов $B$ на $\mathbb{R}^{2}$ (в вещественном случае) или на $\mathbb{C}^{2}$ (в эрмитовом случае), что $\lambda_{1}(B)+$ $\lambda_{2}(B)=0, \lambda_{1}^{2}(B)+\lambda_{2}^{2}(B)=1$, и положим $\mathbb{B}_{2}=\operatorname{conv}\left(\mathbb{S}_{2}\right)$. Тогда $\mathbb{S}_{2}$ есть $(\varepsilon-1)$-мерная сфера, а $\mathbb{B}_{2}$ есть $\varepsilon$-мерный шар, $\mathbb{S}_{2}=\partial \mathbb{B}_{2}$. Пусть $A_{-}$- такой самосопряженный оператор на $\mathbb{R}^{k-1}$ (или на $\mathbb{C}^{k-1}$ ) с простыми собственными числами, что $\lambda_{k-1}\left(A_{-}\right)<-1$, а $A_{+}$- такой самосопряженный оператор на $\mathbb{R}^{n-k-1}$ (или на $\left.\mathbb{C}^{n-k-1}\right)$ с простыми собственными числами, что $\lambda_{1}\left(A_{+}\right)>1$. Тогда $A_{-} \oplus \mathbb{S}_{2} \oplus A_{+}$есть искомое $(\varepsilon-1)$-мерное подмногообразие в $\mathbb{S}$. Действительно, $A_{-} \oplus \mathbb{S}_{2} \oplus A_{+}=\partial\left(A_{-} \oplus \mathbb{B}_{2} \oplus A_{+}\right)$и $\left(A_{-} \oplus \mathbb{B}_{2} \oplus A_{+}\right) \cap\left(\mathbb{S} \backslash \Sigma_{k, k+1}\right)=\left(A_{-} \oplus 0 \oplus A_{+}\right)$; при этом пересечение трансверсально. Следовательно, коэффициент зацепления сферы $A_{-} \oplus \mathbb{S}_{2} \oplus A_{+}$с $\mathbb{S} \backslash \Sigma_{k, k+1}$ равен \pm 1 .

Ограничение расслоения $\mathscr{E}^{k} \rightarrow \Sigma_{k, k+1}$ на $A_{-} \oplus \mathbb{S}_{2} \oplus A_{+}$разлагается в прямую сумму тривиального расслоения и одномерного расслоения над $\mathbb{S}_{2}$, слой которого в точке $B \in \mathbb{S}_{2}$ состоит из собственных векторов, отвечающих собственному числу $\lambda_{1}(B)$. Нетрудно видеть, что отображение, переводящее $B \in \mathbb{S}_{2}$ в прямую, состоящую из собственных векторов с собственным значением $\lambda_{1}(B)$, есть диффеоморфизм пространства $\mathbb{S}_{2}$ и проективной прямой (вещественной или комплексной). Этот диффеоморфизм отождествляет наше одномерное расслоение с тавтологическим расслоением проективной прямой.

Пусть $\delta: H^{i}\left(\Sigma_{k, k+1}\right) \rightarrow H^{i+1}\left(\mathbb{B}, \Sigma_{k, k+1}\right)$ - изоморфизм, индуцированный точной когомологической последовательностью пары $\left(\mathbb{B}, \Sigma_{k, k+1}\right)$. Положим $\Gamma_{k}=\delta \circ \gamma_{k} \in H^{\varepsilon}\left(\mathbb{B}, \Sigma_{k, k+1}\right)$. Значение класса $\Gamma_{k}$ на относительном цикле $\xi \in C_{\varepsilon}\left(\mathbb{B}, \Sigma_{k, k+1}\right)$ равно индексу пересечения этого цикла с $\operatorname{conv}\left(\mathbb{S} \backslash \Sigma_{k, k+1}\right)$.

В заключение этого параграфа приведем явное выражение замкнутой дваформы, представляющей класс $\gamma_{k}$ в эрмитовом случае.

Пусть $A$ - самосопряженный оператор с простыми собственными числами, а $e_{1}, \ldots, e_{n}$ - такой ортонормированный базис, что $A e_{i}=\lambda_{i}(A) e_{i}, i=1, \ldots, n$. Векторы $e_{i}$ определены с точностью до умножения на комплексное число абсолютной величины 1 . Пусть $\langle\cdot, \cdot\rangle$ обозначает эрмитово произведение и $B-$ 
еще один самосопряженный оператор. Нетрудно видеть, что внешний квадрат над $\mathbb{R}$ комплексного числа $\left\langle B e_{i}, e_{j}\right\rangle$ зависит только от $A, B, i, j$, а не от выбора собственных векторов. В частности, $\bigwedge_{\mathbb{R}}^{2}\left\langle d A e_{i}, e_{j}\right\rangle-$ корректно заданная два-форма на пространстве самосопряженных операторов с простыми собственными значениями. Справедлива формула $\bigwedge_{\mathbb{R}}^{2}\left\langle d A e_{i}, e_{j}\right\rangle\left(\partial / \partial B_{1}, \partial / \partial B_{2}\right)=$ $\operatorname{det}_{\mathbb{R}}\left(\left\langle B_{1} e_{i}, e_{j}\right\rangle,\left\langle B_{2} e_{i}, e_{j}\right\rangle\right) ;$ в этой формуле комплексные числа рассматриваются как векторы в $\mathbb{R}^{2}$.

\section{Предложение 3. Форма}

$$
\Omega_{k}=\sum_{i=1}^{k} \sum_{j=k+1}^{n} \frac{1}{\pi\left(\lambda_{i}-\lambda_{j}\right)^{2}} \bigwedge_{\mathbb{R}}^{2}\left\langle d A e_{i}, e_{j}\right\rangle
$$

представляет ограничение класса $\gamma_{k}$ на пространство самосопряженных операторов с простыми собственными числами ${ }^{1)}$.

Набросок доказательства. Нужно доказать, что $\Omega_{k}$ представляет первый класс Чжэня векторного расслоения $\mathscr{E}^{k}$, ограниченного на пространство самосопряженных операторов с простыми собственными числами. Это ограничение расслоения $\mathscr{E}^{k}$ есть прямая сумма одномерных расслоений $\mathscr{L}^{i}, i=1, \ldots, k$, где слой в точке $A$ расслоения $\mathscr{L}^{i}$ есть прямая $L_{A}^{i} \doteq\left\{z \in \mathbb{C}^{n}: A z=\lambda_{i}(A) z\right\}$. Нужно показать, что форма $\Omega_{k}$ представляет класс $\sum_{i=1}^{k} c_{1}\left(\mathscr{L}^{i}\right)$.

Рассмотрим ассоциированное с $\mathscr{L}^{i}$ главное $S^{1}$-расслоение $\mathscr{C}^{i}$; его слой в точке $A$ имеет вид $C_{A}^{i} \doteq\left\{e_{i} \in \mathbb{C}^{n}: e_{i} \in L_{A}^{i},\left|e_{i}\right|=1\right\}$. Пусть $t \mapsto A(t)-$ гладкая кривая в пространстве самосопряженных операторов с простыми собственными числами и $e_{i}(0) \in C_{A(0)}^{i}$. Условие $\left\langle\dot{e}_{i}(t), e_{i}(t)\right\rangle=0$ определяет каноническое поднятие $t \mapsto e_{i}(t)$ кривой $A(\cdot)$ в расслоение $\mathscr{C}^{i}$. Эти поднятия суть параллельные переносы вдоль кривых на базе для подходящей связности на главном расслоении $\mathscr{C}^{i}$. Форма этой связности имеет вид $\operatorname{Im}\left\langle d e_{i}, e_{i}\right\rangle$, где через $\operatorname{Im}$ обозначается мнимая часть комплексного числа.

Внешний дифференциал формы связности $\operatorname{Im}\left\langle d e_{i}, e_{i}\right\rangle$ есть обратный образ формы кривизны связности $R_{i}$. Простое вычисление показывает, что

$$
\left.R_{i}\left(\frac{\partial}{\partial B_{1}}, \frac{\partial}{\partial B_{2}}\right)\right|_{A}=2 \operatorname{Im}\left\langle\frac{\partial e_{i}(A)}{\partial B_{2}}, \frac{\partial e_{i}(A)}{\partial B_{1}}\right\rangle,
$$

где $\partial e_{i}(A) / \partial B=\left.\frac{d}{d t} e_{i}(A+t B)\right|_{t=0}$ и $t \mapsto e_{i}(A+t B)$ параллельно вдоль кривой $t \mapsto A+t B$. Дифференцируя по $t$ уравнение $\left\langle(A+t B) e_{i}(A+t B), e_{j}(A)\right\rangle=\lambda_{i}(A+$ $t B)\left\langle e_{i}(A+t B), e_{j}(A)\right\rangle$, получаем $\left\langle\frac{\partial e_{i}(A)}{\partial B}, e_{j}(A)\right\rangle=\frac{1}{\lambda_{i}(A)-\lambda_{j}(A)}\left\langle B e_{i}(a), e_{j}(A)\right\rangle$ для любого $j \neq i$. Следовательно, $\frac{\partial e_{i}}{\partial B}=\sum_{i \neq j} \frac{1}{\lambda_{i}-\lambda_{j}}\left\langle B e_{i}, e_{j}\right\rangle e_{j}$ и

$$
R_{i}\left(\frac{\partial}{\partial B_{1}}, \frac{\partial}{\partial B_{2}}\right)=\sum_{j \neq i} \frac{2}{\left(\lambda_{i}-\lambda_{j}\right)^{2}} \operatorname{Im}\left(\left\langle B_{2} e_{i}, e_{j}\right\rangle\left\langle B_{1} e_{i}, e_{j}\right\rangle\right) .
$$

1) Форма $\Omega_{k}$ локально ограничена в топологии пространства $\Sigma_{k, k+1}$. Более того, любой двумерный цикл в $\Sigma_{k, k+1}$ гомотопен циклу в пространстве самосопряженных операторов с простыми собственными числами. Следовательно, форма $\Omega_{k}$ действительно представляет класс $\gamma_{k}$. 
В то же время, $\operatorname{Im}\left(z^{1} z^{2}\right)=\operatorname{det}_{\mathbb{R}}\left(z^{2}, z^{1}\right)$ для любых комплексных чисел $z^{1}, z^{2}$. Таким образом, $R_{i}=\sum_{j \neq i} \frac{2}{\left(\lambda_{i}-\lambda_{j}\right)^{2}} \bigwedge_{\mathbb{R}}^{2}\left\langle d A e_{i}, e_{j}\right\rangle$. Складывая, получаем требуемое выражение для формы $\Omega_{k}=\frac{1}{2 \pi} \sum_{i=1}^{k} R_{i}$, представляющей класс $\gamma_{k}$.

\section{§3. Основные результаты}

Рассмотрим фильтрации

$$
M_{k}=\left\{A \in \mathbb{S}: \lambda_{1}(A)=\lambda_{k+1}(A)\right\}, \quad M^{k}=\mathbb{S} \backslash M_{k}, \quad k=0, \ldots, n-1,
$$

пространства $\mathbb{S}$. Нетрудно видеть, что

$$
W_{k} \doteq\left\{A \in \mathbb{S}: \lambda_{k+1}(A)=\lambda_{n}(A)\right\} \subset M^{k}
$$

есть деформационный ретракт пространства $M^{k}$. Ретракция $\phi_{k}: M^{k} \rightarrow W_{k}$ меняет только собственные числа операторов, в то время как собственные векторы остаются неподвижными.

Инволюция $A \mapsto(-A), A \in \mathbb{S}$, переводит $W_{k}$ в $M_{n-k-1}$; следовательно, $M^{k}$ гомотопически эквивалентно $M_{n-k-1}$. Заметим также, что отображение $A \mapsto$ $A-\lambda_{1}(A) I, A \in \mathbb{S}$, индуцирует гомеоморфизм пространства $M_{k}$ и пространства ненулевых неотрицательных самосопряженных операторов ранга $<n-k$, факторизованного по отношению эквивалентности $A \sim \alpha A$ для любого $\alpha>0$.

Везде ниже символ $G r_{k}(m)$ обозначает грассманиан $k$-мерных подпространств пространства $\mathbb{R}^{m}$ или $\mathbb{C}^{m}$.

Теорема 1. $M^{k}$ имеет гомотопический тип пространства Тома некоторого вещественного векторного расслоения над грассманианом $G r_{k}(n-1)$; размерность расслоения равна $k(k+1) / 2+k-1$ в вещественном случае $u k^{2}+2 k-1$ в эрмитовом случае.

Доказательство. Пусть $e-$ вектор единичной длины (в $\mathbb{R}^{n}$ или $\left.\mathbb{C}^{n}\right)$. Положим

$$
G_{k}(e)=\left\{A \in M_{k-1} \cap W_{k}: A e=\lambda_{n}(A) e\right\} .
$$

Тогда $G_{k}(e) \cong G r_{k}(n-1)$. Кроме того, некоторая окрестность подмногообразия $G_{k}(e)$ в $W_{k}$ есть гладкое многообразие и нормальное расслоение к $G_{k}(e)$ в этом многообразии имеет размерность $k(k+1) / 2+k-1$ в вещественном случае и $k^{2}+2 k-1$ в эрмитовом. Это нормальное расслоение разлагается в сумму двух подрасслоений. Первое есть нормальное расслоение подмногообразия $G_{k}(e)$ в $\left\{A \in W_{k}: A e=\lambda_{n}(A) e\right\}$; оно изоморфно расслоению самосопряженных эндоморфизмов с нулевым следом тавтологического расслоения грассманиана $G r_{k}(n-1)$. Второе подрасслоение есть нормальное расслоение подмногообразия $G_{k}(e)$ в $M_{k-1} \cap W_{k}$; оно изоморфно нормальному расслоению подмногообразия $G r_{k}(n-1)$ в $G r_{k}(n)$. Доказываемая теорема есть прямое следствие такого утверждения:

Лемма 1. $W_{k} \backslash G_{k}(e)$ стягиваемо.

Доказательство. Мы стянем $W_{k} \backslash G_{k}(e)$ к точке $-e^{*} \otimes e \in W_{k} \backslash G_{k}(e)$. Наше стягивание переводит $(A, t) \in\left(W_{k} \backslash G_{k}(e)\right) \times[0,1]$ в $\phi_{k}\left(A_{t}\right)$, где $A_{t}=$ $(1-t) A-t e^{*} \otimes e$.

Остается доказать, что стягивание определено корректно, т.е. что $A_{t} \in M^{k}$ и $\phi_{k}\left(A_{t}\right)$ лежит вне $G_{k}(e)$ для любого $t \in[0,1]$. Включение $\phi_{k}\left(A_{t}\right) \in G_{k}(e)$ 
имеет место в том и только том случае, когда $A_{t} \in M_{k-1}$ и $e$ ортогонально подпространству $\left\{x \in X: A_{t} x=\lambda_{1}\left(A_{t}\right) x\right\}$, где $X$ есть $\mathbb{R}^{n}$ (в вещественном случае) или $\mathbb{C}^{n}$ (в эрмитовом случае).

Положим $A^{t}=A-\frac{t}{1-t} e^{*} \otimes e=\frac{1}{1-t} A_{t} ;$ положительный множитель не влияет на кратность собственных чисел, и мы будем работать с $A^{t}$ вместо $A_{t}$. Рассмотрим отдельно два случая.

1. $e \perp\left\{x \in X: A x=\lambda_{i}(A) x, i=1, \ldots, k\right\}$. В этом случае $e-$ собственный вектор оператора $A, A e=\lambda_{n}(A) e$. Следовательно, все $A^{t}$ имеют общие собственные векторы. При этом $n-1$ собственных чисел оператора $A^{t}$ (подсчитанных с учетом кратности) равны собственным числам оператора $A$, а собственное число, отвечающее собственному вектору $e$, монотонно убывает от $\lambda_{n}(A) \mathrm{K}-\infty$, когда $t$ меняется от 0 до 1 . Кроме того, $\lambda_{1}(A) \neq \lambda_{k}(A)$, поскольку $A \notin G_{k}(e)$; следовательно, $A^{t} \in M^{k}$.

Равенство $\lambda_{1}\left(A^{t}\right)=\lambda_{k}\left(A^{t}\right)$ выполняется для некоторого $t \in(0,1]$ в том и только том случае, когда $\lambda_{1}(A)=\lambda_{k-1}(A)$; тогда $A^{t} e=\lambda_{1}\left(A^{1}\right) e$ и $\phi_{k}\left(A^{t}\right) \notin$ $G_{k}(e)$.

2. $e \not \perp\left\{x \in X: A x=\lambda_{i}(A) x, i=1, \ldots, k\right\}$. Ограничение квадратичной формы $x \mapsto\left\langle A^{t} x, x\right\rangle$ на гиперплоскость $e^{\perp}$ не зависит от $t$. Таким образом, из минимаксного принципа для собственных чисел вытекает, что

$$
\lambda_{1}\left(A^{t}\right) \leqslant \lambda_{1}(A) \leqslant \lambda_{2}\left(A^{t}\right) \leqslant \cdots \leqslant \lambda_{k}(A) \leqslant \lambda_{k+1}\left(A^{t}\right) .
$$

Предположим, что $\lambda_{1}\left(A^{t}\right)=\lambda_{k+1}\left(A^{t}\right)$. Следовательно,

$$
\lambda_{1}(A)=\lambda_{k}(A)=\lambda_{1}\left(A^{t}\right)=\min _{|x|=1}\left\langle A^{t} x, x\right\rangle .
$$

В то же время

$$
\left\langle A^{t} x, x\right\rangle=\lambda_{1}(A)-\frac{t}{1-t}\langle e, x\rangle^{2} \quad \forall x \in\left\{x \in X: A x=\lambda_{1}(A) x\right\} .
$$

Мы пришли к противоречию с предположением

$$
e \not \perp\left\{x \in X: A x=\lambda_{1}(A) x\right\}=\left\{x \in X: A x=\lambda_{i}(A) x, i=1, \ldots, k\right\} .
$$

Следовательно, $A_{t} \in M^{k}$.

Теперь предположим, что $\lambda_{1}\left(A^{t}\right)=\lambda_{k}\left(A^{t}\right)$ и $e \perp\left\{x \in X: A^{t} x=\lambda_{1}\left(A^{t}\right) x\right\}$. Тогда

$$
\begin{aligned}
\lambda_{1}\left(A^{t}\right)|x|^{2}=\left\langle A^{t} x, x\right\rangle=\langle A x, x\rangle & =\lambda_{1}(A)|x|^{2} \\
& \forall x \in\left\{x \in X: A^{t} x=\lambda_{i}\left(A^{t}\right) x, i=1, \ldots, k\right\} .
\end{aligned}
$$

Следовательно, $\lambda_{1}(A)=\lambda_{k}(A)$ и

$$
\left\{x \in X: A^{t} x=\lambda_{i}\left(A^{t}\right) x, i=1, \ldots, k\right\}=\left\{x \in X: A x=\lambda_{i}(A) x, i=1, \ldots, k\right\},
$$

что противоречит предположению 2.

Пусть $u_{k} \in H^{\nu_{k}}\left(M^{k}\right)$ - класс Тома нормального расслоения подмногообразия $G_{k}(e)$ в $W_{k}, \nu_{k}=k(k+1) / 2+k-1$ в вещественном случае и $\nu_{k}=k^{2}+2 k-1$ в эрмитовом случае. Пусть $\mathscr{G}_{k}$ - тотальное пространство этого расслоения и $G_{k}(e) \subset \mathscr{G}_{k}$ - его нулевое сечение. Имеют место равенства

$$
\widetilde{H} \cdot\left(M^{k}\right)=H^{\cdot}\left(\mathscr{G}_{k}, \mathscr{G}_{k} \backslash G_{k}(e)\right), \quad H^{\cdot}\left(G_{k}(e)\right)=H^{\cdot}\left(\mathscr{G}_{k}\right),
$$


и когомологическое произведение классов из $\widetilde{H} \cdot\left(M^{k}\right)$ и $H^{\cdot}\left(G_{k}(e)\right)$ есть корректно определенный класс в $\widetilde{H}^{\cdot}\left(M^{k}\right)$. При этом $\xi \mapsto u_{k} \smile \xi, \xi \in H^{\cdot}\left(G_{k}(e)\right)$, есть изоморфизм Тома групп $H\left(G_{k}(e)\right)$ и $\widetilde{H}\left(M^{k}\right)$. Напомним, что $\left.u_{k}\right|_{G_{k}(e)} \in H^{\nu_{k}}\left(G_{k}(e)\right)$ - класс Эйлера расслоения $\mathscr{G}_{k} \rightarrow G_{k}(e)$.

Лемма 2. $\left.u_{k}\right|_{G_{k}(e)}=0$.

Доказательство. Расслоение $\mathscr{G}_{k}$ разлагается в сумму двух подрасслоений, как это было отмечено в доказательстве теоремы 1. Мы покажем, что первое подрасслоение, т.е. расслоение самосопряженных эндоморфизмов с нулевым следом тавтологического расслоения грассманиана, имеет нулевой класс Эйлера. В самом деле, индуцированное расслоение над пространством флагов имеет естественное не обращающееся в нуль сечение: значение такого сечения на некотором флаге есть самосопряженный оператор с предписанными простыми собственными числами, а собственные подпространства суть элементы флага.

Следствие 1. Когомологическое произведение любых двух классов из $\widetilde{H}^{\bullet}\left(M^{k}\right)$ равно нулю.

Доказательство. В силу изоморфизма Тома достаточно показать, что $u_{k} \smile$ $u_{k}=0$, но $u_{k} \smile u_{k}$ есть образ класса $\left.u_{k}\right|_{G_{k}(e)}=0$ при изоморфизме Тома.

Очевидно, что $M^{k}=M^{k-1} \cup \Sigma_{k, k+1}$. Рассмотрим гомоморфизмы

$$
\mathbf{d}_{k}: H^{\cdot}\left(\mathbb{B}, M^{k-1}\right) \rightarrow H^{\cdot}\left(\mathbb{B}, M^{k}\right), \quad k=1, \ldots, n-1,
$$

состоящие в умножении на класс $\Gamma_{k} \in H^{\varepsilon}\left(\mathbb{B}, \Sigma_{k, k+1}\right)$, определенный в конце $\S 2$ :

$$
\mathbf{d}_{k}(\xi)=\Gamma_{k} \smile \xi, \quad \xi \in H^{\cdot}\left(\mathbb{B}, M^{k-1}\right) .
$$

Напомним, что $\varepsilon=2$ в вещественном случае и $\varepsilon=3$ в эрмитовом случае.

Теорема 2. Последовательность

$$
0 \rightarrow H^{\cdot}(\mathbb{B}) \stackrel{\mathbf{d}_{1}}{\rightarrow} H^{\cdot}\left(\mathbb{B}, M^{1}\right) \stackrel{\mathbf{d}_{2}}{\rightarrow} \ldots \stackrel{\mathbf{d}_{n-2}}{\longrightarrow} H^{\cdot}\left(\mathbb{B}, M^{n-2}\right) \stackrel{\mathbf{d}_{n-1}}{\rightarrow} H^{\cdot}(\mathbb{B}, \mathbb{S}) \rightarrow 0
$$

является точной.

Доказательство. Мы проведем вычисления только в вещественном случае; эрмитов вариант разбирается аналогично.

Прежде всего заметим, что $\Gamma_{k} \smile \Gamma_{k-1}=0$. В самом деле, $\Gamma_{k} \smile \Gamma_{k-1}$ есть элемент группы $H^{4}\left(\mathbb{B}, \Sigma_{k-1, k} \cup \Sigma_{k, k+1}\right)=H^{4}\left(\mathbb{S}, \Sigma_{k-1, k} \cup \Sigma_{k, k+1}\right)$, но

$$
\mathbb{S} \backslash\left(\Sigma_{k-1, k} \cup \Sigma_{k, k+1}\right)=\left\{A \in \mathbb{S}: \lambda_{k-1}(A)=\lambda_{k+1}(A)\right\}
$$

- алгебраическое подмножество коразмерности 5 в $\mathbb{S}$ (см. предложение 1). Следовательно, $\mathbf{d}_{k} \circ \mathbf{d}_{k-1}=0$ и последовательность (1) образует коцепной комплекс. Требуется доказать тривиальность когомологий этого комплекса.

Рассмотрим пространства

$$
\Sigma_{1, k, k+1} \doteq\left\{A \in \mathbb{S}: \lambda_{1}(A) \neq \lambda_{k}(A) \neq \lambda_{k+1}(A)\right\}=M^{k-1} \cap \Sigma_{k, k+1} .
$$

Заметим, что $\phi_{k}\left(\Sigma_{k, k+1}\right)=\Sigma_{k, k+1} \cap W_{k}$ и $\Sigma_{k, k+1} \cap W_{k}$ - деформационный ретракт пространства $\Sigma_{k, k+1}$. Аналогично, $\phi_{k}\left(\Sigma_{1, k, k+1}\right)=\Sigma_{1, k, k+1} \cap W_{k}$ и $\Sigma_{1, k, k+1} \cap$ $W_{k}$ - деформационный ретракт пространства $\Sigma_{1, k, k+1}$. 
Отображение $A \mapsto \operatorname{span}\left\{x \in \mathbb{R}^{n}: A x=\lambda_{i}(A) x, i=1, \ldots, k\right\}$ превращает $\Sigma_{k, k+1} \cap W_{k}$ в локально тривиальное расслоение над $G r_{k}(n)$ со слоем в точке $E \in G r_{k}(n)$, состоящим из всех таких самосопряженных операторов $A: E \rightarrow E$, что $\sum_{i=1}^{k} \lambda_{i}(A) \leqslant 0, \sum_{i=1}^{k} \lambda_{i}^{2}(A)+\frac{1}{n-k}\left(\sum_{i=1}^{k} \lambda_{i}(A)\right)^{2}=1$; такие операторы однозначно продолжаются до (нормализованных) операторов из $W_{k}$. Таким образом, слой представляет собой шар размерности $k(k+1) / 2-1=\nu_{k-1}+1$.

Далее, $\left(\Sigma_{k, k+1} \backslash \Sigma_{1, k, k+1}\right) \cap W_{k}$ есть сечение расслоения $\Sigma_{k, k+1} \cap W_{k} \rightarrow G r_{k}(n)$, причем значение этого сечения в точке $E \in G r_{k}(n)$ - скалярный оператор на $E$, «центр шара». Следовательно, «сферическое расслоение»

$$
\left\{A \in \Sigma_{k, k+1} \cap W_{k}: \sum_{i=1}^{k} \lambda_{i}(A)=0\right\}
$$

с общим слоем $S^{\nu_{k-1}}$ есть гомотопический ретракт пространства $\Sigma_{1, k, k+1}$. Пусть

$$
\begin{aligned}
& S_{E}^{\nu_{k-1}} \doteq\left\{A \in \Sigma_{k, k+1} \cap W_{k}: \sum_{i=1}^{k} \lambda_{i}(A)=0, A x_{i}=\lambda_{i}(A) x_{i},\right. \\
&\left.x_{i} \in E \backslash\{0\}, i=1, \ldots, k\right\}
\end{aligned}
$$

- слой сферического расслоения в точке $E$.

Лемма 3. Ограничение $\left.u_{k-1}\right|_{S_{E}^{\nu_{k-1}}}$ класса $u_{k-1} \in H^{\nu_{k-1}}\left(M^{k-1}\right)$, индуцированное включением

$$
M^{k-1} \supset \Sigma_{1, k, k+1} \supset S_{E}^{\nu_{k-1}},
$$

есть образующая группъ $H^{\nu_{k-1}}\left(S_{E}^{\nu_{k-1}}\right)$.

Доказательство. Значение класса Тома $u_{k-1}$ на цикле $S_{E}^{\nu_{k-1}}$ равно индексу пересечения цикла $\phi_{k-1}\left(S_{E}^{\nu_{k-1}}\right)$ с подмногообразием $G_{k-1}(e)$ в $M^{k-1} \cap W_{k-1}$. Очевидно, что это значение не зависит от $E$. Выберем такое $E$, что $e \not \perp E$. Тогда пересечение подмногообразий $\phi_{k-1}\left(S_{E}^{\nu_{k-1}}\right)$ и $G_{k-1}(e)$ трансверсально и состоит из одной точки $A_{0}$, определяемой условиями

$$
A_{0} \in G_{k-1}(e), \quad\left\{x \in \mathbb{R}^{n}: A_{0} x=\lambda_{1}\left(A_{0}\right) x\right\}=e^{\perp} \cap E .
$$

Следствие 2. Пусть $v_{k-1}=\left.u_{k-1}\right|_{\Sigma_{1, k, k+1}}$. Тогдакольио $H^{\cdot}\left(\Sigma_{1, k, k+1}\right)$ есть свободный модуль над кольцом $H^{\cdot}\left(G r_{k}(n)\right)$ с базисом 1, $v_{k-1}$. При этом $v_{k-1} \smile$ $v_{k-1}=0$.

Доказательство. Структура модуля порождена структурой расслоения $\Sigma_{1, k, k+1} \cap W_{k} \rightarrow G r_{k}(n)$. Тот факт, что модуль свободный, следует из леммы 3 и теоремы Лере-Хирша. Равенство $v_{k-1} \smile v_{k-1}=0$ вытекает из равенства $u_{k-1} \smile u_{k-1}=0$ (см. следствие 1$)$.

Лемма 4. Включения $M^{k-1} \subset M^{k} u \Sigma_{k, k+1} \subset M^{k}$ индуцируют нулевой гомоморфизм приведенных групп когомологий.

Доказательство. Имеет место включение $\phi_{k}\left(M^{k-1}\right) \subset M^{k} \backslash G_{k}(e)$. Следовательно, $M^{k-1}$ содержится в стягиваемом подмножестве пространства $M^{k}$ и 
ограничение на $M^{k-1}$ превращает любой класс когомологий из $\widetilde{H}\left(M^{k}\right)$ в нулевой класс. Рассмотрим теперь включения

$$
\Sigma_{1, k, k+1} \subset \Sigma_{k, k+1} \subset M^{k} .
$$

Из следствия 2 вытекает, что включение $\Sigma_{1, k, k+1} \subset \Sigma_{k, k+1} \cong G r_{k}(n)$ индуцирует инъективный гомоморфизм $H^{\cdot}\left(\Sigma_{k, k+1}\right) \rightarrow H^{\cdot}\left(\Sigma_{1, k, k+1}\right)$. В то же время $\phi_{k}\left(\Sigma_{1, k, k+1}\right) \subset M^{k} \backslash G_{k}(e)$. Следовательно, композиция индуцированных вложениями гомоморфизмов

$$
\widetilde{H}^{\cdot}\left(M^{k}\right) \rightarrow \widetilde{H}^{\cdot}\left(\Sigma_{k, k+1}\right) \rightarrow \widetilde{H}^{\cdot}\left(\Sigma_{1, k, k+1}\right)
$$

равна нулю. Стало быть, гомоморфизм $\widetilde{H}^{\cdot}\left(M^{k}\right) \rightarrow \widetilde{H}^{\cdot}\left(\Sigma_{k, k+1}\right)$ также равен нулю.

Пусть $X \subset \mathbb{S}$ - такое открытое подмножество в $\mathbb{S}$, что его дополнение есть окрестностный деформационный ретракт; через $\hat{\delta}: \widetilde{H}^{i}(X) \rightarrow H^{i+1}(\mathbb{B}, X)$ мы обозначаем естественный изоморфизм, индуцированный точной последовательностью пары $\mathbb{B}, X$.

Рассмотрим теперь точную последовательность Майера-Вьеториса пары $\Sigma_{k, k+1}, M^{k-1}$

$$
\ldots H^{i-1}\left(M^{k}\right) \rightarrow H^{i-1}\left(\Sigma_{k, k+1}\right) \oplus H^{i-1}\left(M^{k-1}\right) \stackrel{\theta}{\rightarrow} H^{i-1}\left(\Sigma_{1, k, k+1}\right) \stackrel{d}{\rightarrow} H^{i}\left(M^{k}\right) \ldots,
$$

а также ее относительный вариант

$$
\ldots H^{i}\left(\mathbb{B}, M^{k}\right) \rightarrow H^{i}\left(\mathbb{B}, \Sigma_{k, k+1}\right) \oplus H^{i}\left(\mathbb{B}, M^{k-1}\right) \stackrel{\theta}{\rightarrow} H^{i}\left(\mathbb{B}, \Sigma_{1, k, k+1}\right) \stackrel{d}{\rightarrow} H^{i+1}\left(\mathbb{B}, M^{k}\right) \ldots
$$

Естественный изоморфизм $\hat{\delta}$ устанавливает изоморфизм этих двух длинных точных последовательностей. Более того, из леммы 4 следует, что длинная точная последовательность разбивается на короткие:

$$
0 \rightarrow H^{i-1}\left(\Sigma_{k, k+1}\right) \oplus H^{i-1}\left(M^{k-1}\right) \stackrel{\theta}{\rightarrow} H^{i-1}\left(\Sigma_{1, k, k+1}\right) \stackrel{d}{\rightarrow} H^{i}\left(M^{k}\right) \rightarrow 0,
$$

и аналогичное утверждение справедливо для относительного варианта.

Лемма 5. Пусть $\xi \in H^{*}\left(\Sigma_{k, k+1}\right), \eta \in H^{*}\left(M^{k-1}\right)$. Тогда равенство $\hat{\delta} \xi \smile \hat{\delta} \eta$ $=0$ эквивалентно включению $\left(\left.\left.\xi\right|_{\Sigma_{1, k, k+1}} \smile \eta\right|_{\Sigma_{1, k, k+1}}\right) \in \operatorname{im} \theta$.

Доказательство. Предложение из добавления А влечет за собой равенство

$$
\hat{\delta} \xi \smile \hat{\delta} \gamma=\delta \circ d\left(\left.\left.\xi\right|_{\Sigma_{1, k, k+1}} \smile \eta\right|_{\Sigma_{1, k, k+1}}\right) .
$$

Утверждение доказываемой леммы теперь следует из точности последовательности (3) и того факта, что $\hat{\delta}-$ изоморфизм.

Следующий шаг - описание $\operatorname{im} \theta$. Для заданных $\xi \in H^{\cdot}\left(\Sigma_{k, k+1}\right), \eta \in H^{*}\left(M^{k-1}\right)$ получаем

$$
\theta(\xi \oplus \eta)=\left.\xi\right|_{\Sigma_{1, k, k+1}}-\left.\eta\right|_{\Sigma_{1, k, k+1}} .
$$

Согласно следствию 2 , ограничение $\left.H^{\cdot}\left(\Sigma_{k, k+1}\right) \rightarrow H^{\cdot}\left(\Sigma_{k, k+1}\right)\right|_{\Sigma_{1, k, k+1}}$ инъективно и

$$
H^{\cdot}\left(\Sigma_{1, k, k+1}\right)=\left.H^{\cdot}\left(\Sigma_{k, k+1}\right)\right|_{\Sigma_{1, k, k+1}} \oplus\left(\left.v_{k-1} \smile H^{\cdot}\left(\Sigma_{k, k+1}\right)\right|_{\Sigma_{1, k, k+1}}\right) .
$$


Напомним, что $M^{k-1}$ имеет гомотопический тип пространства Тома векторного расслоения над $G_{k-1}(e) \subset M^{k-1}$ с классом Тома $u_{k-1} \in H^{\nu_{k-1}}\left(M^{k-1}\right)$. Рассмотрим отображение $\varrho_{k}: H^{\cdot}\left(G_{k-1}(e)\right) \rightarrow H^{\cdot}\left(\Sigma_{k, k+1}\right)$, где

$$
\left.v_{k-1} \smile \varrho_{k}(\zeta)\right|_{\Sigma_{1, k, k+1}}=\left.\pi_{v}\left(u_{k-1} \smile \zeta\right)\right|_{\Sigma_{1, k, k+1}} \quad \forall \zeta \in H^{\cdot}\left(G_{k-1}(e)\right) .
$$

Тождество (4) однозначно определяет $\varrho_{k}$. Более того, отображение $\varrho_{k}$ инъективно и

$$
\operatorname{im} \theta=\left.H^{\cdot}\left(\Sigma_{k, k+1}\right)\right|_{\Sigma_{1, k, k+1}} \oplus\left(\left.v_{k-1} \smile \operatorname{im} \varrho_{k}\right|_{\Sigma_{1, k, k+1}}\right) .
$$

Пространство $\Sigma_{k, k+1}$ имеет гомотопический тип грассманиана $G r_{k}(n)$, а $G_{k-1}(e)$ отождествляется с грассманианом $\left\{F \in G r_{k-1}(n): F \subset e^{\perp}\right\}=G r_{k-1}(n-1)$. Мы явным образом вычислим отображение $\varrho_{k}$ в базисах, заданных клетками Шуберта грассманианов.

В последующих вычислениях мы отождествляем многообразие

$$
\Sigma_{k, k+1} \cap M_{k-1} \cap W_{k}=\left(\Sigma_{k, k+1} \backslash \Sigma_{1, k, k+1}\right) \cap W_{k}
$$

с грассманианом $G r_{k}(n)$, причем $A \in \Sigma_{k, k+1} \cap M_{k-1} \cap W_{k}$ отождествляется с подпространством $\left\{x \in \mathbb{R}^{n}: A x=\lambda_{1}(A) x\right\}$. Пространство $\Sigma_{k, k+1} \cap M_{k-1} \cap$ $W_{k}$, очевидно, есть гомотопический ретракт пространства $\Sigma_{k, k+1}$. В частности, $H^{\cdot}\left(\Sigma_{k, k+1}\right)=H^{\cdot}\left(G r_{k}(n)\right)$.

Пусть $e_{1}=e, e_{2}, \ldots, e_{n}$ - ортогональный базис пространства $\mathbb{R}^{n}$. Отвечающие этому базису замкнутые клетки Шуберта в $G r_{k}(n)$ суть циклы, задающие аддитивный базис в гомологиях $H .\left(G r_{k}(n)\right)$ (см. добавление В). Рассматривается также двойственный базис Шуберта в когомологиях $H^{\cdot}\left(G r_{k}(n)\right)$. Клетки Шуберта размерности $r \geqslant 0$ находятся во взаимно однозначном соответствии с разбиениями числа $r$ на не более чем $k$ целых положительных слагаемых, каждое из которых не превосходит $n-k$.

Аналогично, клетки Шуберта, отвечающие базису $e_{2}, \ldots, e_{n}$ пространства $e^{\perp}=\mathbb{R}^{n-1}$, определяют базис Шуберта в когомологиях $H^{\cdot}\left(G r_{k-1}(n-1)\right)=$ $H^{\cdot}\left(G_{k-1}(e)\right)$. Элементы размерности $r$ этого базиса находятся во взаимно однозначном соответствии с разбиениями числа $r$ на менее чем $k$ целых положительных слагаемых, каждое из которых не превосходит $n-k$.

Лемма 6. Отображение $\varrho_{k}: H^{\cdot}\left(G_{k-1}(e)\right) \rightarrow H^{\cdot}\left(G r_{k}(n)\right)$ переводит элемент базиса Шуберта в когомологиях $H^{*}\left(G_{k-1}(e)\right)$, отвечающий некоторому разбиению на менее чем $k$ слагаемых, в элемент базиса Шуберта в когомологиях $H^{*}\left(G r_{k}(n)\right)$, отвечающий тому же самому разбиению!

Доказательство. Мы рассмотрим сопряженное отображение $\varrho_{k}^{*}: H .\left(G r_{k}(n)\right)$ $\rightarrow H .\left(G_{k-1}(e)\right)$. Требуется доказать, что $\varrho_{k}^{*}$ переводит в нуль классы Шуберта в $H .\left(G r_{k}(n)\right)$, отвечающие разбиениям на в точности $k$ слагаемых, в то время как классы, отвечающие разбиениям на меньше чем $k$ слагаемых, переходят в классы Шуберта в $H .\left(G_{k-1}(e)\right)$, отвечающие тем же разбиениям.

Пусть $C \subset G r_{k}(n)$ - некоторый цикл Шуберта и $[C]-$ его класс гомологий. Положим $S_{C}^{\nu_{k-1}}=\bigcup_{E \in C} S_{E}^{\nu_{k-1}}$, см. (2). Тогда $\varrho_{k}^{*}[C]-$ класс гомологий пересечения $\phi_{k-1}\left(S_{C}^{\nu_{k-1}}\right)$ с $G_{k-1}(e)=G r_{k-1}(n-1)$. Иными словами, отображение $\varrho_{k}^{*}$ по существу определяется многозначным отображением $\mathfrak{r}_{k}: G r_{k}(n)-\rightarrow G_{k-1}(e)$, где $\mathfrak{r}_{k}(E)=\phi_{k-1}\left(S_{E}^{\nu_{k-1}}\right) \cap G_{k-1}(e), E \in G r_{k}(n)$. 
Нетрудно видеть, что $\mathfrak{r}_{k}(E)=\left\{F \in G r_{k-1}(n-1): F \subset E \cap e^{\perp}\right\}$. В частности, отображение $\mathfrak{r}_{k}$ однозначно на $\left\{E \in G r_{k}(n): E \not \perp e\right\} ;$ если $E \not \perp e$, то $\mathfrak{r}_{k}(E)=$ $E \cap e^{\perp}$. Однозначное отображение $F \mapsto(F+\mathbb{R} e), F \in G r_{k-1}(n-1)$, является обратным к $\mathfrak{r}_{k}$.

Пусть $\mathfrak{d}$ - некоторый символ Шуберта для $G r_{k}(n)$, начинающийся с единицы. Тогда $\mathfrak{r}_{k}\left(S c_{k}^{\mathfrak{d}}(n)\right)=S c_{k-1}^{\mathfrak{d}^{\prime}}(n-1)$, причем символ $\mathfrak{d}^{\prime}$ получается из символа $\mathfrak{d}$ удалением первой единицы. В самом деле, $e \in E$ для любого $E \in S c_{k}^{\mathfrak{d}}(n)$, и требуемое равенство легко следует из определений.

Предположим теперь, что $\mathfrak{d}$ - некоторый символ Шуберта для $G r_{k}(n)$, начинающийся с нуля. Покажем, что $\mathfrak{r}_{k}\left(S c_{k}^{\mathfrak{d}}(n)\right)$ содержится в объединении клеток Шуберта размерности, строго меньшей, чем размерность клетки $S c_{k}^{\mathfrak{d}}(n)$. Этот факт завершает доказательство леммы 6 .

Пусть $F \in S c_{k}^{\mathfrak{d}}(n)$ и $\hat{F} \in \mathfrak{r}_{k}\left(S c_{k-1}^{\hat{\mathfrak{d}}}(n-1)\right)$. Напомним, что

$$
\begin{aligned}
d_{i}^{\mathfrak{d}}=\min \left\{j: \operatorname{dim}\left(E_{j} \cap F\right)=i\right\}, & i=1, \ldots, k, \\
d_{i}^{\hat{\mathfrak{d}}}=\min \left\{j: \operatorname{dim}\left(E_{j+1} \cap \hat{F}\right)=i\right\}, & i=1, \ldots, k-1,
\end{aligned}
$$

где $E_{j}=\operatorname{span}\left\{e_{1}, \ldots, e_{j}\right\}, j=1, \ldots, n$. В то же время,

$$
\operatorname{dim}\left(E_{j} \cap F_{j}\right)-1 \leqslant \operatorname{dim}\left(E_{j} \cap \hat{F}_{j}\right) \leqslant \operatorname{dim}\left(E_{j} \cap F_{j}\right) ;
$$

следовательно, $d_{i}^{\mathfrak{d}} \leqslant d_{i}^{\hat{\mathfrak{d}}}+1 \leqslant d_{i+1}^{\mathfrak{d}}$. Кроме того, $d_{1}^{\mathfrak{d}}>1$, поскольку символ $\mathfrak{d}$ начинается с 0 . Мы получаем

$$
\begin{aligned}
\operatorname{dim} S c_{k-1}^{\hat{\mathfrak{d}}}(n-1) & =\sum_{i=1}^{k-1}\left(d_{i}^{\hat{\mathfrak{d}}}-i\right) \\
& =\sum_{i=1}^{k-1}\left(\left(d_{i}^{\hat{\mathfrak{d}}}+1\right)-(i+1)\right) \leqslant \sum_{i=2}^{k}\left(d_{i}^{\mathfrak{d}}-i\right)<\operatorname{dim} S c_{k}^{\mathfrak{d}}(n) .
\end{aligned}
$$

Лемма 7. Пусть $w \in H^{1}\left(G_{k}(n)\right)$ - первый класс Штифеля-Уитни тавтологического расслоения и $\xi \in \operatorname{im} \varrho_{k}$. Включение $(w \smile \xi) \in \operatorname{im} \varrho_{k}$ справедливо в том и только том случае, когда $\xi$ есть сумма классов Шуберта, отвечающих разбиениям на строго менъше чем $k-1$ слагаемых.

Доказательство. Пусть $\Pi_{j}$ - линейная оболочка классов Шуберта, отвечающих разбиениям на в точности $j$ слагаемых, $j=0,1, \ldots, k$, и $\xi-$ класс Шуберта, отвечающий разбиению $a_{1}+\cdots+a_{j}$. Из формулы Пьери (см. добавление В) вытекает, что разность произведения $w \smile \xi$ и класса Шуберта, отвечающего разбиению $1+a_{1}+\cdots+a_{j}$, лежит в $\Pi_{j}$. С другой стороны, согласно лемме $6, \operatorname{im} \varrho_{k}=\bigoplus_{j=0}^{k-1} \Pi_{j}$.

Теперь мы готовы к вычислению $\operatorname{ker} \mathbf{d}_{k}$ и, таким образом, завершению доказательства теоремы 2. Пусть $\xi \in H^{*}\left(M^{k-1}\right)$; тогда $\xi=\hat{\delta}\left(u_{k-1} \smile \zeta\right)$ для некоторого $\zeta \in H^{\cdot}\left(G_{k-1}(e)\right)$, определенного единственным образом. Мы имеем

$$
\mathbf{d}_{k}(\xi)=\hat{\delta}\left(\gamma_{k}\right) \smile \hat{\delta}\left(u_{k-1} \smile \zeta\right),
$$


где $\gamma_{k} \in H^{1}\left(\Sigma_{k, k+1}\right)$ - класс, определенный в 2 . Согласно лемме 5, равенство $\mathbf{d}_{k}(\xi)=0$ имеет место в том и только том случае, когда

$$
\left.\left.\left(u_{k-1} \smile \zeta\right)\right|_{\Sigma_{1, k, k+1}} \smile \gamma_{k}\right|_{\Sigma_{1, k, k+1}}=\left(\left.v_{k-1} \smile\left(\varrho_{k}(\zeta) \smile \gamma_{k}\right)\right|_{\Sigma_{1, k, k+1}}\right) \in \operatorname{im} \theta .
$$

Далее, $G_{k}(n)=\Sigma_{k, k+1} \cap M_{k-1} \cap W_{k}$ есть гомотопический ретракт пространства $\Sigma_{k, k+1}$, а $\left.\gamma_{k}\right|_{G r_{k}(n)}$ - первый класс Штифеля-Уитни тавтологического расслоения грассманиана $G r_{k}(n)$, т. е. $\left.\gamma_{k}\right|_{G r_{k}(n)}=w$ (см. предложение 2). Из равенства (5) теперь следует, что $\mathbf{d}_{k}(\xi)=0$ в том и только том случае, когда $\left(\varrho_{k}(\zeta) \smile w\right) \in \operatorname{im} \varrho_{k}$.

Из леммы 7 и инъективности $\varrho_{k}$ вытекает, что $\operatorname{dim} k e r \mathbf{d}_{k}$ равно количеству разбиений на не более чем $k-2$ натуральных слагаемых, каждое из которых не превосходит $n-k$. Иными словами, dim ker $\mathbf{d}_{k}=\left(\begin{array}{l}n-2 \\ k-2\end{array}\right)$. В то же время, изоморфизмы $H^{\cdot}\left(\mathbb{B}, M^{k-1}\right) \cong \widetilde{H} \cdot\left(M^{k-1}\right) \cong H^{\cdot}\left(G r_{k-1}(n-1)\right)$ влекут за собой равенство $\operatorname{dim} H^{\cdot}\left(\mathbb{B}, M^{k-1}\right)=\left(\begin{array}{l}n-1 \\ k-1\end{array}\right)$. Теперь воспользуемся «треугольником Паскаля» $\left(\begin{array}{l}n-1 \\ k-1\end{array}\right)=\left(\begin{array}{l}n-2 \\ k-2\end{array}\right)+\left(\begin{array}{l}n-2 \\ k-1\end{array}\right)$ и получим

$\operatorname{dim} H^{\cdot}\left(\mathscr{B}, M^{k-1}\right)=\operatorname{dim} \operatorname{ker} \mathbf{d}_{k}+\operatorname{dim} \operatorname{ker} \mathbf{d}_{k+1}$.

\section{А. Одно свойство когомологического произведения}

Пусть $M$ - симплициальный комплекс, а $X \subset M-$ его подкомплекс; обозначим символом $\delta_{X}: H^{*}(X) \rightarrow H^{*+1}(M, X)$ связывающий гомоморфизм в точной когомологической последовательности пары $M, X$. Пусть $Y \subset M-$ еще один подкомплекс и $d: H^{*}(X \cap Y) \rightarrow H^{*+1}(X \cup Y)-$ связывающий гомоморфизм в точной когомологической последовательности Майера-Вьеториса пары $X, Y$.

Предложение. Пусть $\xi \in H^{*}(X), \eta \in H^{*}(Y) ;$ тогда

$$
\delta_{X} \xi \smile \delta_{Y} \eta=\delta_{X \cup Y} \circ d\left(\left.\left.\xi\right|_{X \cap Y} \smile \eta\right|_{X \cap Y}\right) .
$$

Доказательство. Положим $\zeta=d\left(\left.\left.\xi\right|_{X \cap Y} \smile \eta\right|_{X \cap Y}\right)$. Пусть $x$ и $y-$ коциклы, представляющие когомологические классы $\xi$ и $\eta$. Представителем класса $\zeta$ служит любой коцикл $z$, такой, что

$$
\left.z\right|_{X}=\delta u,\left.\quad z\right|_{Y}=\delta v,\left.\quad u\right|_{X \cap Y}-\left.v\right|_{X \cap Y}=\left.\left.x\right|_{X \cap Y} \smile y\right|_{X \cap Y}
$$

для некоторых коцепей $u, v$. Можно действовать следующим образом: продолжить $x$ и $y$ до коцепей $\hat{x}$ и $\hat{y}$ на $X \cup Y$ и положить $z=\hat{x} \smile \delta \hat{y}$. Тогда условия $(\mathrm{A})$ выполняются для $u=\left.(-1)^{\operatorname{dim} x} x \smile \hat{y}\right|_{X}, v=0$ и мы получаем $\delta z=\delta \hat{x} \smile \delta \hat{y}$.

\section{В. Клетки Шуберта}

Клетки Шуберта задают структуру клеточных комплексов на грассманианах. Эти клетки индексируются символами Шуберта. Символ Шуберта $\mathfrak{d}$ для $G r_{k}(n)$ есть последовательность нулей и единиц, содержащая ровно $k$ единиц и $n-k$ нулей. Общее число символов для $G r_{k}(n)$ (т. е. число клеток клеточного комплекса) равно $\left(\begin{array}{l}n \\ k\end{array}\right)$. Обозначим через $d_{i}^{\mathfrak{d}}$ номер $i$-й единицы в последовательности; тогда $1 \leqslant d_{1}^{\mathfrak{d}}<\cdots<d_{k}^{\mathfrak{d}} \leqslant n$. 
Мы рассматриваем одновременно вещественный и комплексный случаи. Пусть $e_{1}, \ldots, e_{n}$ - некоторый базис пространства $\mathbb{R}^{n}$ в вещественном и пространства $\mathbb{C}^{n}$ в комплексном случае. Положим

$$
E_{i}=\operatorname{span}\left\{e_{1}, \ldots, e_{i}\right\}, \quad i=1, \ldots, n .
$$

Клетка Шуберта $S c_{k}^{\mathfrak{d}}(n)$ определяется следующим образом:

$$
S c_{k}^{\mathfrak{d}}(n)=\left\{F \in G r_{k}(n): \operatorname{dim}\left(F \cap E_{d_{i}^{\mathfrak{o}}}\right)=i, \operatorname{dim}\left(F \cap E_{d_{i}^{\mathrm{o}}-1}\right)=i-1\right\} .
$$

Имеется взаимно однозначное соответствие между символами Шуберта для $G r_{k}(n)$ и разбиениями неотрицательных целых чисел на не более чем $k$ целых положительных слагаемых, каждое из которых не превосходит $n-k$. Слагаемые, отвечающие символу $\mathfrak{d}$, суть числа нулей слева от каждой из единиц, входящих в символ. Иными словами, слагаемые суть ненулевые члены последовательности $\left(d_{i}^{\mathfrak{d}}-i\right), i=1, \ldots, k$.

Размерность клетки Шуберта, отвечающей разбиению числа $r$, равна $r$ в вещественном и $2 r$ в комплексном случае. Таким образом, справедливо равенство

$$
\operatorname{dim} S c_{k}^{\mathfrak{d}}(n)=\epsilon \sum_{i=1}^{k}\left(d_{i}^{\mathfrak{d}}-i\right),
$$

где $\epsilon=1$ в вещественном и $\epsilon=2$ в комплексном случае. Замыкание $\overline{S c_{k}^{\mathfrak{d}}(n)}$ есть цикл с коэффициентами в $\mathbb{Z}_{2}$ в вещественном и цикл с коэффициентами в $\mathbb{Z}$ в комплексном случае; эти циклы называются ииклами Шуберта. В обоих случаях классы гомологий циклов Шуберта образуют аддитивный базис полной группы гомологий грассманиана $G r_{k}(n)$ (с коэффициентами в $\mathbb{Z}_{2}$ в вещественном и с коэффициентами в $\mathbb{Z}$ в комплексном случае). Более того, в комплексном случае группы гомологий свободны.

Двойственный базис полной группы когомологий грассманиана $G r_{k}(n)$ называется базисом Шуберта. Напомним, что элементы этого базиса размерности $r$, т. е. $r$-мерные классы Шуберта, находятся во взаимно однозначном соответствии с разбиениями числа $r$ на не более чем $k$ натуральных слагаемых, каждое из которых не превосходит $n-k$.

Характеристические классы Штифеля-Уитни (в вещественном случае) и Чжэня (в комплексном случае) суть классы Шуберта, отвечающие разбиениям на единицы. В частности, класс Штифеля-Уитни $w_{1}$ в вещественном и класс Чжэня $c_{1}$ в комплексном случае отвечают единственному «разбиению» числа 1.

Имеется полезная формула Пьери, вычисляющая когомологическое произведение класса Шуберта, отвечающего разбиению с одним слагаемым $a$, и класса Шуберта, отвечающего произвольному разбиению $b_{1}+\cdots+b_{j}$, где $b_{1} \leqslant \cdots \leqslant b_{j}$. Произведение равно сумме всех классов Шуберта размерности $a+\sum_{i=1}^{j} b_{i}$, отвечающих таким разбиениям $c_{0}+c_{1}+\cdots+c_{j}$, что $b_{i-1} \leqslant c_{i} \leqslant b_{i}, i=1, \ldots, j$, $b_{0}=0$.

Подробности см. в [4, гл. 5] и [3, гл. 1.5].

\section{ЛитератУРА}

[1] В. И. Арнольд, Моды и квазимоды, Функц. анализ и его прил., 6:2 (1972), 12-20.

[2] V. I. Arnold, Remarks on eigenvalues and eigenvectors of Hermitian matrices, Berry phase, adiabatic connections and quantum Hall effect, Selecta Math., 1:1 (1995), 1-19. 
[3] Ф. Гриффитс, Дж. Харрис, Принципы алгебрачческой геометрии, тт. 1, 2, Мир, M., 1982.

[4] Дж. Милнор, Дж. Сташеф, Характеристические классы, Мир, М., 1979.

[5] M. Shapiro, A. Vainshtein, Stratification of Hermitian matrices and the Alexander mapping, C. R. Acad. Sci., Sér. I, 321:12 (1995), 1599-1604.

SISSA, Триест

Математический институт им. В. А. Стеклова РАН e-mail: agrachevaa@gmail.com
Поступило в редакцию

15 июля 2011 г. 